\title{
Experimental and physics based study of the Schottky Barrier Height inhomogeneity and associated traps affecting 3C-SiC-on-Si Schottky Barrier Diodes
}

\author{
A. Arvanitopoulos, F. Li, M. R. Jennings, S. Perkins, K. N. Gyftakis, Phil Mawby, M. Antoniou, N. Lophitis
}

\begin{abstract}
The ability of cubic phase (3C-) Silicon Carbide (SiC) to grow heteroepitaxially on Silicon ( $\mathrm{Si}$ ) substrates (3C$\mathrm{SiC}-o n-\mathrm{Si}$ ) is an enabling feature for cost-effective Wide Bandgap devices and homogeneous integration with $\mathrm{Si}$ devices. In this paper, the authors evaluated $3 \mathrm{C}-\mathrm{SiC}$-on-Si Schottky Barrier Contacts by fabricating and testing non-freestanding lateral Schottky Barrier Diodes (LSBD). To gain a deep physical insight of the complex carrier transport phenomena that take place in this material, advanced Technology Computer Aided Design (TCAD) models were developed which allowed accurately matching of measurements with simulations. The models incorporate the device geometry, an accurate representation of the bulk material properties, and complex trapping/de-trapping and tunnelling phenomena which appear to affect the device performance. The observed non-uniformities of the Schottky Barrier Height (SBH) were successfully modelled through the incorporation of interfacial traps. The combination of TCAD with fabrication and measurements enabled the identification of trap profiles and pin their influence on the electrical performance of $3 \mathrm{C}$-SiC-on-Si LSBD. The effect of temperature was studied by engaging the identified trap profiles and calculating the occupation distribution of electrons in $3 \mathrm{C}-\mathrm{SiC}$ at elevated temperature. The investigation constitutes an imperative knowledge step towards the development of devices that take advantage of $3 \mathrm{C}-\mathrm{SiC}$ material properties.
\end{abstract}

Keywords-Schottky contacts, inhomogeneity, traps, $\mathrm{SiC}, 3 \mathrm{C}$ SiC-on-Si, TCAD

\section{INTRODUCTION}

$\mathrm{T}$ he demands of modern applications in power electronics make the Silicon- ( $\mathrm{Si}$ ) based power devices lag, mainly due to the moderate critical electric field value of the material [1], [2]. On the other hand, wide bandgap (WBG) semiconductors, like Silicon Carbide (SiC), promise advanced properties [3]-[5]. The 3C-SiC, also referred to as $\beta-\mathrm{SiC}$, is the only form of $\mathrm{SiC}$ with a cubic crystal structure, similar to $\mathrm{Si}[6]$. In consequence, its ability to heteroepitaxially grow on large $\mathrm{Si}$ substrates (3C-SiC-on$\mathrm{Si}$ ) meets the low-cost requirement and, concurrently,

A. Arvanitopoulos, Faculty of Engineering, University of Nottingham, Nottingham, UK (e-mail: a.e.arvanitopoulos@ieee.org).

F. Li, School of Engineering, University of Warwick, Coventry, UK (e-mail: f.li.3@warwick.ac.uk).

M. R. Jennings, College of Engineering, Swansea University, Swansea, UK (e-mail: m.r.jennings@swansea.ac.uk).

S. Perkins, Faculty of EEC at Coventry University, Coventry, UK (email: perkin19@uni.coventry.ac.uk).

K. N. Gyftakis, School of Engineering, University of Edinburgh, UK, (e-mail: k.n.gyftakis@ieee.org).

P. Mawby, School of Engineering, University of Warwick, Coventry, UK (e-mail: p.a.mawby@warwick.ac.uk)

M. Antoniou, School of Engineering, University of Warwick, Coventry, UK (e-mail: marina.antoniou@warwick.ac.uk).

N. Lophitis, Faculty of Engineering, University of Nottingham, Nottingham, UK (e-mail: neo.lophitis@ nottingham.ac.uk). encourages possible integration with $\mathrm{Si}$ devices [7], [8]. The induced isotropic properties of $3 \mathrm{C}-\mathrm{SiC}$ due to its cubic structure, along with its refractory nature, the superior electron mobility at high temperatures and the high thermal conductivity also emphasize its importance [9]-[11].

The larger energy band gap of 3C-SiC allows higher doping and thinner layers for power devices with given blocking capabilities. Thus, resulting in much lower specific on-resistance, particularity for unipolar devices, like Schottky Barrier Diodes (SBDs). Nonetheless, these diodes do not reach their full potential due to an observed excessive leakage current [12]. This deteriorates their performance in both forward and reverse bias conditions and hence, currently obstructs them from commercialization. Notably, when 3C$\mathrm{SiC}$ is grown on $\mathrm{Si}$, various types of bulk traps are formed in the epitaxial layers [13]-[17], due to lattice and thermal mismatches at the heterointerface [18]. The electrical activity of such extended defects in 3C-SiC-on-Si, like stacking faults (SFs) and micro-twins [19], is a major concern for functional power devices. The carriers tend to flow preferentially through these defects, leading to high leakages and low breakdown voltages. In [20] anti-phase boundaries (APBs) were identified to be the main responsible for the enhanced leakage current under reverse bias polarization and both APBs and SFs were shown to work as preferential current paths responsible for the reduced turn-on voltage under forward bias. In addition, the deposition of a metal on any semiconductor leads to the formation of supplementary electronic states at the Schottky interface [21]. The presence of such a variety of traps affects the metal / 3C-SiC interfaces and the formation of Schottky barriers in particular [22]

Furthermore, reported inhomogeneous features of the Schottky contact can essentially add to the resulted leakage current before the onset of forward conductivity or while in blocking mode [23]. In the literature there have been various attempts to model such features. In [24]-[26] a simplified approach assumed the contact divided in two parts, each one characterized by an independent SBH value. This introduced the concept of parallel conduction in which two or more discrete barrier heights are operating in parallel at the Metal / $\mathrm{SiC}$ interfaces [27]-[31]. In a similar manner, in [32]-[34] the SBH values were modelled with a Gaussian distribution resulting in a non-uniform behaviour of the Schottky contact under voltage stress. A very well established model was developed by Tung to describe the inhomogeneity observed in fabricated Schottky contacts and has emerged as the most complete method to incorporate parallel conduction methodology [35], [36]. According to Tung's theory, the inhomogeneous behaviour of the SBH is attributed to potential fluctuations at the Schottky interface, but no assumptions are made on the origins of this observed inhomogeneity [25]. 
These fluctuations are considered as a localized phenomenon and spatially limited on small portions of the contact, called patches [37]. The Tung's model is applied by determining the values of fitting parameters from a modified Thermionic Emission (TE) equation based on the suggestion that neighbouring patches interact. However, questions have been raised about the physical interpretation of the fitting parameters of this model for diodes that widely deviate from the ideal TE behaviour $(\eta>1.21)$, irrespective of the material system [25], [38]. Such obtained highly unphysical parameters indicate that the source of the significant degree of non-ideality in these SBDs is possibly extrinsic in nature, i.e. traps and/or process induced impurities.

It is of interest that both the degradation of the electrical performance and the observed inhomogeneous of a Schottky contact have been linked with the presence of traps in fabricated SBDs [39]-[44]. However, the characterization of these traps' and their overall influence on the barrier height value of 3C-SiC-on-Si SBDs are still mostly unknown and unclear [26].

In this paper, 3C-SiC-on-Si lateral SBDs (LSBDs) were fabricated to perform an experimental investigation of the traps affecting the carrier transport mechanisms. Based on validated 3C-SiC material models [45], [46], an advanced Technology Computer Aided (TCAD) model is developed allowing for the identification of the trap profiles in the diode. These trap profiles include both bulk traps and Schottky interface states. The impact to the Schottky Barrier Height (SBH) due to the presence of these traps was revealed with simulations and fully justifies the high leakage current observed in the fabricated diodes. Furthermore, the proposed model attributes non-uniformities of the SBH to additional trap profiles at the Schottky interface. The multiple interfacial trap profiles are differentiated by distinct spatial distributions on the active area of the contact.

The simplicity of LSBD structure allows for gaining insights of the traps' impact on leakage current. In this work the existence of the traps is correlated with complex trapping/de-trapping and tunnelling phenomena that enhance the recombination/ generation mechanism of carriers, depending on the bias. The attention is in the forward bias region of the device operation, rather than the reverse, which constrains any electric field in the vicinity of the contacts being studied. This reduces the possibility of interaction and thus interference from possible defects in e.g. the $\mathrm{Si} / 3 \mathrm{C}-\mathrm{SiC}$ interface. The analysis methodology, including the modelled influence of the traps is not limited to 3C-SiC-on-Si SBDs but can be also applied for any SBD, particularly in cases where high ideality factor is observed.

\section{DEVICE FABRICATION AND CHARACTERISATION}

Circular and rectangular/striped contacts have been fabricated on a $3 \mathrm{C}-\mathrm{SiC}$-on-Si wafer. The grown $3 \mathrm{C}-\mathrm{SiC}$ layer is $4.28 \mu \mathrm{m}$ thick and of $5 \times 10^{16} \mathrm{~cm}^{-3}$ Nitrogen (n) doped. The Si substrate utilized is Boron (B) doped and features p-type conductivity characteristics with a resistivity value of 5-10 $\Omega \cdot \mathrm{cm}$. High concentration of Nitrogen $(\mathrm{n}+)$ with doping around $5 \times 10^{20} \mathrm{~cm}^{-3}$ and depth of $300 \mathrm{~nm}$ was achieved with ion implantation in selective regions. It was activated at $1350^{\circ} \mathrm{C}$ for 2 hours. The active doping level estimated to be $\sim 7 \times 10^{19}$ $\mathrm{cm}^{-3}$ with sheet resistance $\sim 3 \mathrm{~m} \Omega . \mathrm{cm}$ [47]. A layer of $500 \mathrm{~nm}$ was grown to passivate the $\mathrm{SiC}$ surface. Ohmic contacts were obtained on these implanted regions by etching the $\mathrm{SiO}_{2}$ and depositing a Ti30nm/Ni100nm bilayer and annealed at $1000^{\circ} \mathrm{C}$ for $1 \mathrm{~min}$. The Schottky contacts were formed by evaporating $200 \mathrm{~nm}$ of Platinum (Pt) and no further treatment was applied. No trench isolation was attempted because the target has been to maintain a simple process flow and reduce the risk of introducing fixed charges on etched surfaces and thus extra leakage current from potentially non optimised complex process steps. There is however a $\mathrm{SiO}_{2}$ layer $(500 \mathrm{~nm})$ passivation layer between the anode and cathode contact and between adjacent devices. In addition, the distance between adjacent devices is much larger compared with the anodecathode distance to reduce possible interference.

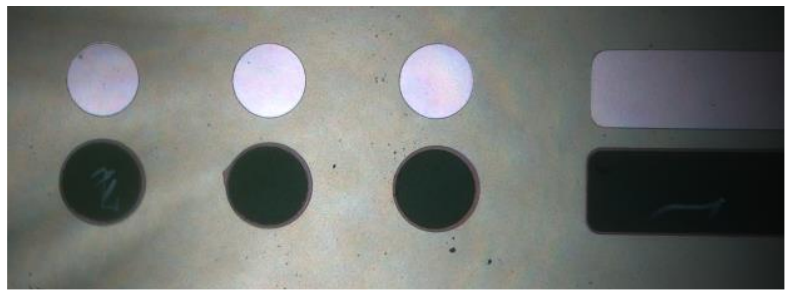

(a) Circular and striped LSBDs fabricated on 3C-SiC-on-Si wafers. The dark coloured contacts are ohmic, whilst the brightest are Pt-Schottky
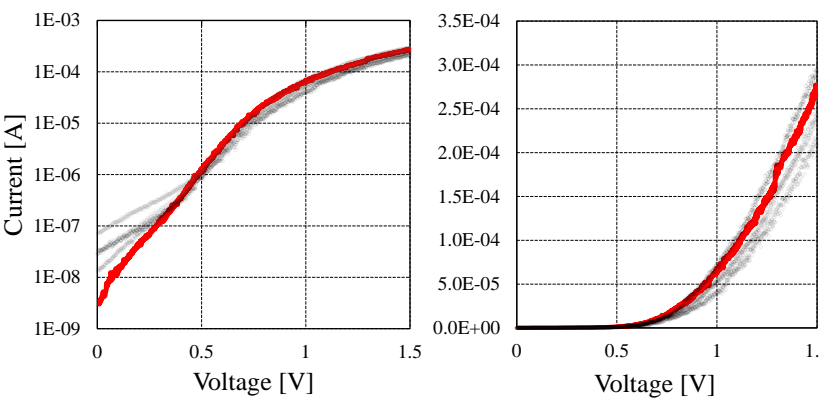

(b) Forward characteristics of striped LSBDs featuring $100 \mu \mathrm{m}$ contacts.

The device with the highlighted characteristics (red line) features the lowest ideality factor and has been chosen for further analysis.

Fig. 1 LSBDs fabricated on 3C-SiC-on-Si wafers with representative characteristics.

In Fig. 1(a), a sample of the fabricated LSBDs on 3C$\mathrm{SiC}(100) / \mathrm{Si}(100)$ is shown. The dark coloured contacts are the ohmic contacts, whilst the brightest correspond to the PtSchottky ones. To obtain diode variants, two design variables were introduced in the design split. The diameter and/or the width accounted as the first variable for the circular and the rectangular contacts correspondingly. The circular shaped LSBDs featured $\varnothing 100 \mu \mathrm{m}$ for the smaller contacts and $\emptyset 150 \mu \mathrm{m}$ for the larger ones. Accordingly, small rectangular contacts were of $100 \mu \mathrm{m}$ width, whereas the large ones were of $150 \mu \mathrm{m}$ width. The lateral separation between the ohmic and Schottky contacts was the second design variable with values ranging 30,35 and $40 \mu \mathrm{m}$.

The Keysight B1505A parametric analyser [48] was utilized for the characterization process. Thereafter, the ideality factor $(\eta)$ was calculated from the I-V measurements for each LSBD. Most devices were found to feature large $\eta$ values. In addition, the ideality factor of the diodes was not constant rather it demonstrated a varying value with applied forward bias. The stripped LSBDs have been chosen for further analysis because their geometry is closer to that of two infinitely long parallel plates. This reduces the impact of geometry on the results and the analysis can be done with two dimensional rather than three dimensional models. The small sized striped diodes achieved the lower $\eta$ values compared to 
those with larger dimensions and their forward I-V characteristics are shown in Fig.1(b). The device with the lowest $\eta$ achieved is shown in Fig. 1(b) with red highlight and has been selected for TCAD modelling and analysis through simulations.

\section{Device Modelling Methodology}

The cross-section of the investigated as-deposited $\mathrm{Pt} / 3 \mathrm{C}$ SiC-on-Si LSBD is illustrated in Fig. 2. The 3C-SiC material layer is $4.28 \mu \mathrm{m}$ thick and $5 \times 10^{15} \mathrm{~cm}^{-3}$ Nitrogen $(\mathrm{N})$ doped The Schottky metal is Platinum with a workfunction of $q \Phi_{M}=5.65 \mathrm{eV}$ [49]. The Schottky and ohmic contacts are laterally separated by $40 \mu \mathrm{m}$. The Si substrate is considered lowly doped and of opposite conductivity type compared to the drift, according to the wafer specifications. An area factor has been utilized to accurately represent the diode in the third dimension. Towards accurate simulations, additional to the Thermionic Emission (TE), the barrier lowering due to image force and the barrier tunnelling physical mechanisms are also considered. The TCAD 3C-SiC material physical models used in this work were previously developed and validated in [45].
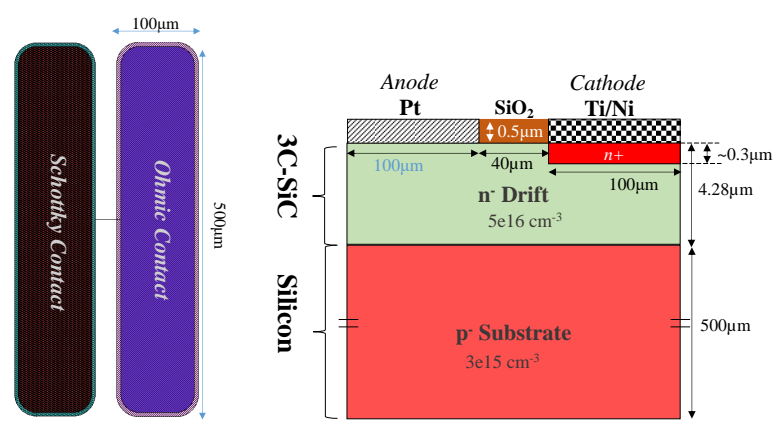

Fig. 2 The top view (left side) and cross-section (right side) of the 3C$\mathrm{SiC}(100) / \mathrm{Si}(100)$ LSBD characterised and modelled.

\section{A. Modelling the carrier transport mechanisms.}

To ascertain the full impact of traps with physics-based simulations it is key to consider the correct carrier transport mechanisms. Thermionic Emission (TE), is not adequate to replicate the complex nature of carriers' transport under the influence of traps, particularly when the ideality factor is significantly larger than unity [50]. This is because it assumes that the transmission probability for energies lower than the expected barrier height is negligible. Field Emission (FE) corresponds to current conduction through the barrier by tunnelling and it turns to be increasingly important when the barrier becomes thin. Another significant carrier mechanism is the Thermionic Field Emission (TFE), which combines thermionic emission and tunnelling. Further, the TrapAssisted Tunnelling (TAT) is a non-local field emission process realized in two steps and could also contribute to the current given the presence of traps. Barrier lowering (BL) is a physical phenomenon during which the effective barrier of the Schottky contact, and thus the level of current, is modified under the influence of electric field. This phenomenon is usually considered when an external reverse bias is applied and therefore high electric field is induced. Nonetheless, BL can occur even at zero or positive voltage bias and it can be particularly evident when studying the subthreshold characteristics. It manifests when a thin layer of high concentration donor states residing on (or very close to) the metal/semiconductor interface become ionized, forming a layer of positive charge. Under these conditions a thin high electric field layer is formed which bends the bands, effectively reducing the barrier height as illustrated in Fig. 3(a). Under such conditions BL can have a strong influence on the current and being able to account for this phenomenon is critical when using physics-based simulations to analyse real measurements. The conduction mechanisms and the influence of BL are diagrammatically shown in Fig. 3(b). They are all accounted for in this work.

In order to replicate the measurements with simulations, an advanced TCAD model is proposed, which suggests the inclusion of trap profiles and their sub-sequent effect on the carrier transport mechanisms. Furthermore, the sub-threshold region of the forward $\log (\mathrm{I})-\mathrm{V}$ is directly linked to the quality of the semiconductor material [51]. This can be interpreted with the existence of traps both at the Schottky interface and the drift. The observed variations of the value of $\eta$ with the applied voltage signifies that the effect of these traps depends on the bias level and, thus, the Fermi level $\left(E_{F}\right)$ position [52]. Although this specific LSBD featured the lowest ideality factor values of the batch, these values of $\eta \approx 3$ are still larger than unity, as shown in Fig. 4, indicating the significant effect from the presence of traps on the subthreshold current. Focusing on the forward I-V characteristics of the fabricated diode, simulations were performed with the modelled structure in Fig. 2. Matching the simulations with the experiments, within the bias region, allows for the identification of existing bulk traps in the drift of the investigated 3C-SiC-on-Si LSBD.

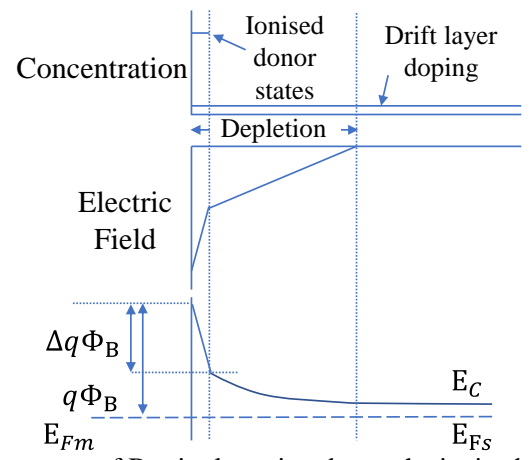

(a) The phenomenon of Barrier lowering due to the ionized donor states at the interface between $3 \mathrm{C}-\mathrm{SiC}$ and the Schottky contact.
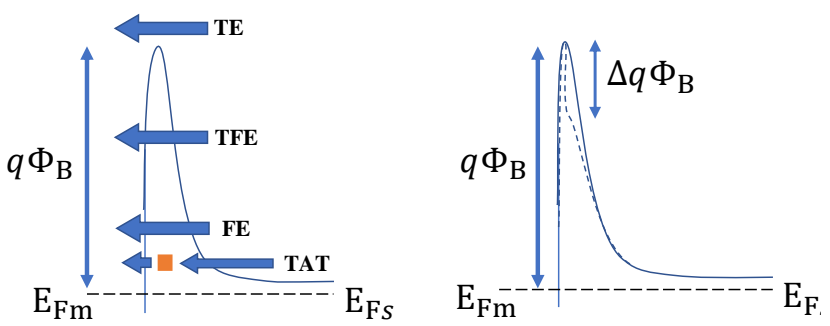

(b) Representation of conduction mechanisms and the influence of barrier lowering.

Fig. 3 Conduction methods at the metal-semiconductor contact

\section{TRAPS IDENTIFICATION}

\section{A. Bulk traps}

Bulk traps are considered deep levels, with their origin assumed to be defects related to the $3 \mathrm{C}-\mathrm{SiC} / \mathrm{Si}$ hetero interface. Focusing on the bias region, a correlation process between measurements and Finite Element Analysis (FEA), utilizing the device model in Fig. 2, identified the properties of the bulk traps likely to be present in the $3 \mathrm{C}-\mathrm{SiC}$ drift. These are modelled to cover the full drift region, reaching the 


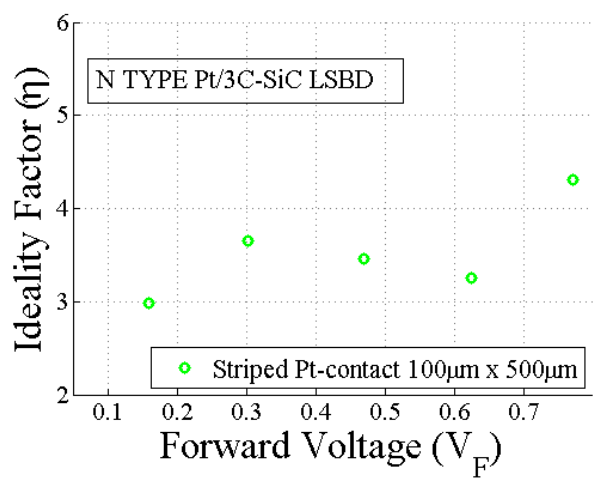

Fig. 4 The calculated $\eta$ values with a step of 60 measurements of the applied forward voltage for the fabricated and characterized 3C-SiC-on-Si LSBD (modelled in Fig. 2).

contact. The determined properties are presented in Table I and their behaviour as deep acceptors resembles intrinsic Silicon Vacancies $\left(V_{S i}\right)$ defects [53]. Such defects are common in $3 \mathrm{C}$-SiC-on-Si, originating from the out-diffusion of the $\mathrm{Si}$ atoms to form the $\mathrm{SiC}$ layer [7]. The activation energy of these bulk traps, given in Table I, indicates high probability of occupation with majority carriers even at zero bias. Therefore, the reduced population of free electrons in the $3 \mathrm{C}-\mathrm{SiC}$ drift in thermal equilibrium $\left(n_{0}\right)$ results in an effective Fermi level $\left(E_{F}^{e f f}\right)$ to be considered according to (1), which is illustrated in the band diagram in Fig. 5. Notably, in thermal equilibrium the relative position of the $E_{F}^{e f f}$ at the Schottky interface $\left(\left.E_{F}^{e f f}\right|_{I T}\right)$ changes to a lower energy.

$$
n_{0}=n_{i} \exp \left(\frac{E_{F}-E_{g} / 2}{k T}\right)
$$

The effect on the forward I-V characteristics from the inclusion of the identified bulk traps in the TCAD model is illustrated in Fig. 6. Focusing on the on-state region of the forward I-V characteristics, shown in Fig. 6(a), in the absence of bulk traps, the predicted I-V characteristics have a significantly smaller differential resistance (green dotted line vs red solid line) when compared to the measurements. The inclusion of deep acceptors, resembling $V_{S i}$, increase the ndrift resistance by capturing majority electrons, thus impacting the differential resistance. Their inclusion results in achieving the same slope $\left(R_{\text {differential }}^{-1}\right)$ for both the simulated characteristics and the measurements. A second observation is that the barrier height predicted with simulations features a larger value compared to the characterized diode. In Schottky junctions, the barrier height, or the build-in potential $\left(q V_{b i}\right)$, is the potential barrier that obstructs free electrons flow from the semiconductor to the Schottky metal side. In consequence, the actual devices start to conduct earlier. The inclusion of further bulk traps compared to those already added does not affect the barrier height but significantly affects the differential resistance. The bulk traps are act uniformly all over the epitaxy layer, also reaching the vicinity of the Schottky contact. As shown in Fig. 6(b), they thus affect the sub-threshold I-V curve too but the impact they have is marginal - the sub-threshold current predicted is still at least ten orders of magnitude lower than the measurements. This suggests the presence of additional states at the Schottky junction, which magnify the contribution of the TFE, FE and TAT conduction mechanisms over the TE one. In Fig. 6(b), the inability of the characterized fabricated diodes (open cycle symbols) to comply with the predicted TE theory (dashed line) in the subthreshold region is also highlighted in a log-scale representation of Fig. 6(a).

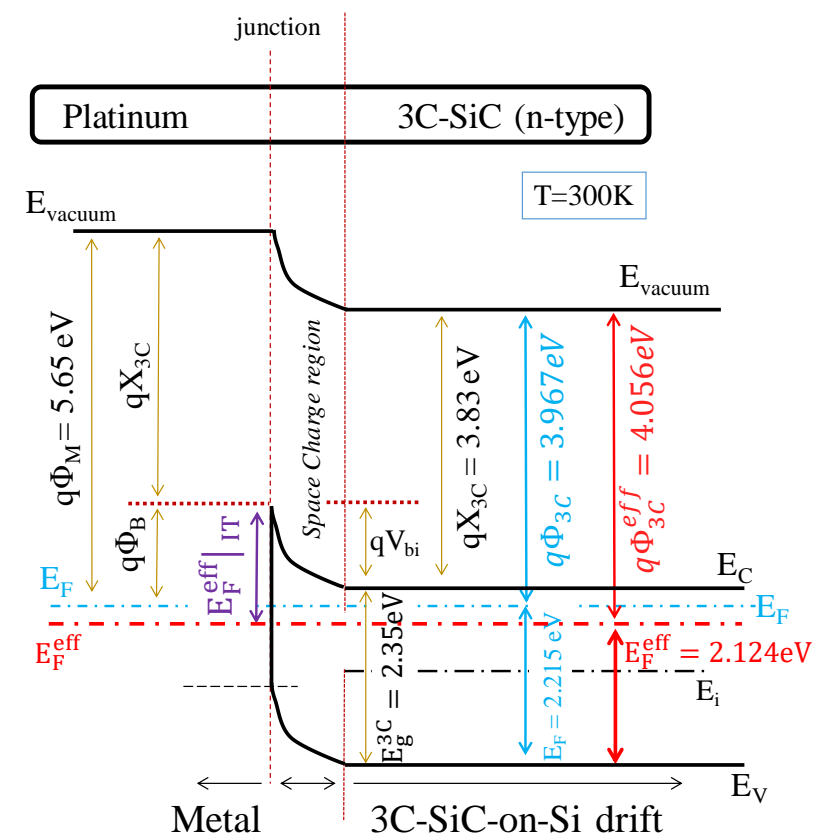

Fig. 5 Band diagram of LSBD at zero bias and $\mathrm{T}=300 \mathrm{~K}$. The deep bulk acceptor levels, resembling $V_{S i}$, are highly likely to capture majority carriers, and thus, alter the energy of the Fermi level from an initial $E_{F}$ into $E_{F}^{e f f}$ in the band diagram.
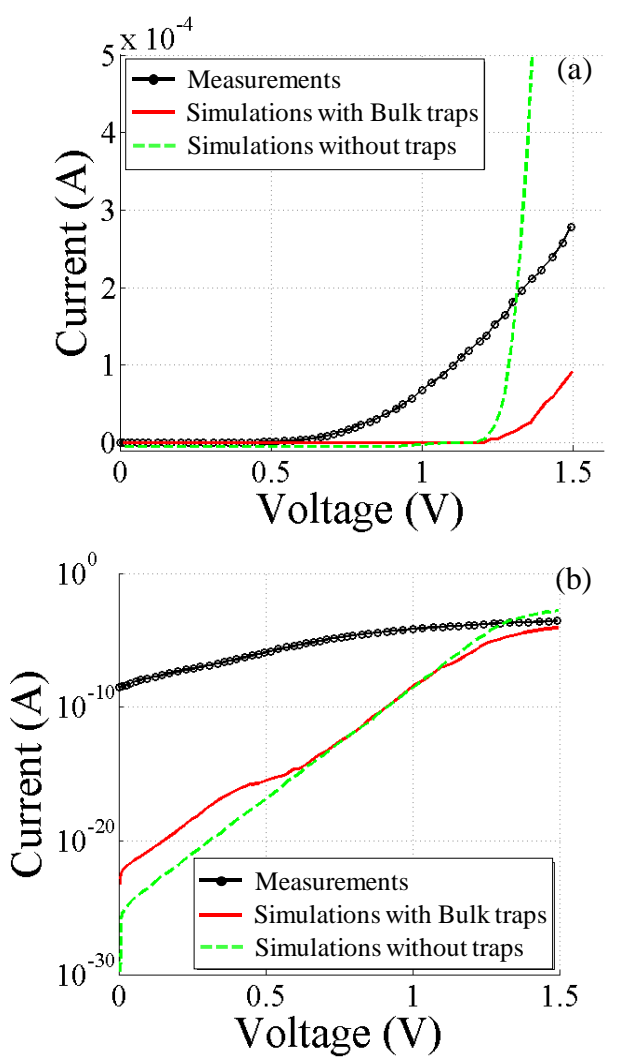

Fig. 6 (a) The inclusion of the deep acceptor traps from Table I contributes on achieving a simulated on-resistance similar to the one of the characterized diode. (b) The same in log-scale. 


\section{B. Interface traps: Model A - spatially uniform interfacial traps acting over the full length of the Schottky contact}

The states at the Schottky interface are modelled with uniform energetic distributions expanding over a rage of activation values. This attributes the model with a voltage-dependent activation capable of emulating the presence of a varying amount of fixed charges at the interface. In turn, the enhanced electric fields uniformly alter the electrical behaviour of the SBH [54], [55]. The properties of interfacial traps were determined through FEM and are presented in Table I. The interface traps of Model A comprise two energetic distributions, one of donor-like states and another of acceptorlike states. The definition of these energetic distributions defines a range of activation energies within which states can behave both as acceptors and donors. These states, between $E_{C}^{3 C-S i C}-0.1 \mathrm{eV}$ and $E_{C}^{3 C-S i C}-0.4 \mathrm{eV}$, are called amphoteric [56], [57]. An illustration of how these states are energetically distributed at the interface between $\mathrm{Pt}$ and 3C$\mathrm{SiC}$-on-Si is shown in Fig. 7. Initially it is assumed that these traps are homogeneously distributed (spatially) throughout the full length of the Schottky metal.

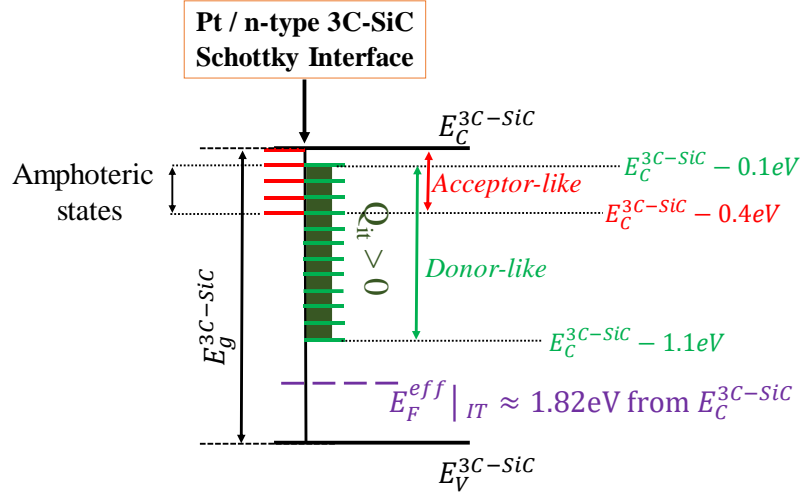

Fig. 7 The energetic distributions of the identified traps' model A at the Schottky interface. The position of the $\left.E_{F}^{e f f}\right|_{I T}$ decides which interfacial states are highly likely to be occupied depending on their type.

Table I. Identified trap profiles in the fabricated and investigated 3CSiC-on-Si LSBD.

\begin{tabular}{|c|c|c|c|c|}
\hline \multirow{2}{*}{\multicolumn{2}{|c|}{ Trap specification }} & \multirow{2}{*}{$\begin{array}{c}\text { Type / } \\
\text { Concentration }\end{array}$} & \multicolumn{2}{|c|}{ Distributions } \\
\hline & & & Energetic & Spatial \\
\hline$\stackrel{5}{3}$ & $\begin{array}{c}\text { Deep } \\
\text { levels due } \\
\text { to } 3 C \text { - } \\
\text { SiC/Si } \\
\text { hetero- } \\
\text { interface } \\
\text { to model } \\
\text { the } \\
V_{S i} \text { defects }\end{array}$ & $\begin{array}{c}4.85 \times 10^{16} \mathrm{~cm}^{-3} \\
\text { of } \\
\text { Acceptors }\end{array}$ & $\begin{array}{l}\text { Single Level } \\
\text { Activation } \\
\text { Energy } \\
E^{A}=0.5 \mathrm{eV} \\
\left(\text { from } E_{V}^{3 C}\right)\end{array}$ & $\begin{array}{l}\text { Uniform } \\
\text { covering } \\
\text { all the } \\
\text { drift } 3 \mathrm{C} \text { - } \\
\mathrm{SiC} \\
\text { layer, } \\
\text { reaching } \\
\text { the } \\
\text { contact }\end{array}$ \\
\hline \multirow{2}{*}{ 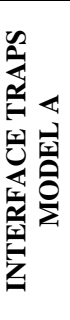 } & $\begin{array}{l}\text { Schottky } \\
\text { contact } \\
\text { interfacial } \\
\text { defects } \\
\text { recultino }\end{array}$ & $\begin{array}{c}8 \times 10^{18} \mathrm{~cm}^{-3} \\
\text { of } \\
\text { Donor-like } \\
\text { states }\end{array}$ & $\begin{array}{c}\text { Uniform Band } \\
E_{\text {Mid }}=0.6 \mathrm{eV} \\
E_{\text {Sig }}=1.0 \mathrm{eV} \\
\left(\text { from } E_{C}^{3 C}\right)\end{array}$ & \multirow{2}{*}{$\begin{array}{l}\text { Uniform } \\
\text { along all } \\
\text { the } \\
\text { contact } \\
\text { in the } \\
\text { lateral } \\
\text { direction }\end{array}$} \\
\hline & $\begin{array}{l}\text { in a } \\
\text { specific } \\
\text { effect on } \\
\text { the barrier } \\
\text { height }\end{array}$ & $\begin{array}{c}2 \times 10^{18} \mathrm{~cm}^{-3} \\
\text { of } \\
\text { Acceptor-like } \\
\text { states }\end{array}$ & $\begin{array}{c}\text { Uniform Band } \\
E_{\text {Mid }}=0.2 \mathrm{eV} \\
E_{\text {Sig }}=0.4 \mathrm{eV} \\
\left(\text { from } E_{C}^{3 C}\right)\end{array}$ & \\
\hline
\end{tabular}

The calculated relative position of the $E_{F}^{e f f}$ at the interface decides which of these states are ionized, and, thus, highly likely to become occupied by a carrier. The donor-like states, at the interface, energetically located above the $E_{F}^{e f f}$ are able to capture a hole emulating fixed positive charge. The formation of positive charge at the Schottky interface induces band bending, altering the SBH value. This effect is shown in Fig. 8, where the interface traps Model A, with the energetic distributions shown in Fig. 7, results in a reduced potential barrier $\left(q V_{b i}^{e f f}\right)$ for electrons. The dotted $E_{C}$ line in Fig. 8 corresponds to the initial band bending due to the space charge region formation in thermal equilibrium. This is identical to the case illustrated at the band diagram in Fig. 5.

Fig. 9 depicts the subthreshold region and how the various mechanisms contribute to the subthreshold current when traps (Table I-Model A) exist at the Schottky interface. It is clear, that TE alone underestimates the subthreshold current, and more importantly it cannot accurately predict the shape of the $\log (\mathrm{I})-\mathrm{V}$ characteristic. This observation is in agreement with the large calculated ideality factor values of the investigated LSBD. The traps' Model A defines an energetic distribution of donor-like states of high concentration. When ionized they enhance the electric field of the space charge region which lowers the effective barrier (Fig 3 right). In turn, the BL allows for more majority carriers to cross the barrier through TFE and FE, making them significant contributors to the sub-threshold current.

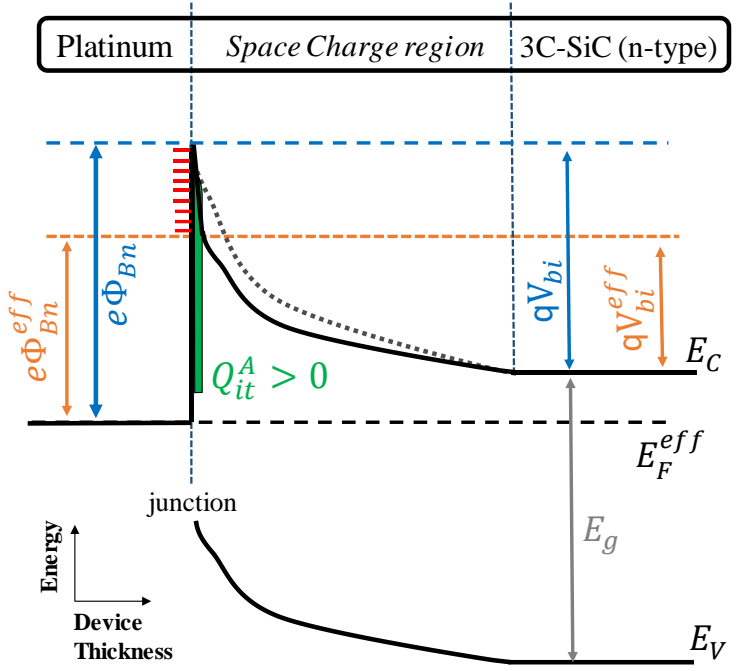

Fig. 8 The effect of the interfacial traps Model A, in Fig. 7, on the SBH in equilibrium. The dashed line corresponds to the initial $\mathrm{E}_{\mathrm{C}}$ band calculated in Fig. 5.

A part of the FE electrons (contribute to the current of the investigated 3C-SiC-on-Si SBD by completing a direct transition to the conduction band $\left(E_{C}\right)$ of the Metal. The rest of the majority electrons tunnelling through the Schottky interface, between $\mathrm{Pt}$ and $3 \mathrm{C}-\mathrm{SiC}$, recombine with holes at the valence band $\left(E_{V}\right)$ of the metal. For recombination to happen both types of carriers need to be present. The metal system is assumed to be a pool of electrons and holes, thus contributing to holes. Both the direct FE and recombination at the Schottky interface due to tunnelling have a strong impact on ideality factor, deviating its value towards values greater than unity.

The amphoteric interfacial states, in Fig 7, correspond to traps which can either behave as donors or acceptors. Whilst the external bias level (indicated by $\left.E_{F}^{e f f}\right|_{I T}$ at the Schottky interface) is below these amphoteric states, they are able to capture holes. With the increasing forward bias, the energy bands move upwards and these states will eventually drop below the $\left.E_{F}^{e f f}\right|_{I T}$. In this case, they act as acceptors and are able to capture electrons. The latter decreases the number of 
the majority carriers that can contribute to the sub-threshold current, or locally increases the resistance. Hence, the energetic distribution of the acceptor-like states in Model A, in Table I, has been specified so to balance the strong effect from the donor-like states of Model A. Interestingly, the acceptor-like states demonstrated a negligible contribution to the TAT recombination. This is attributed to the properties of the donor-like distribution of Model A, which energetically expands up to the $\mathrm{E}_{\mathrm{C}}$. The resultant strong band bending at the Schottky interface, therefore, encourages the direct FE rather than the indirect TAT transition for the electrons.

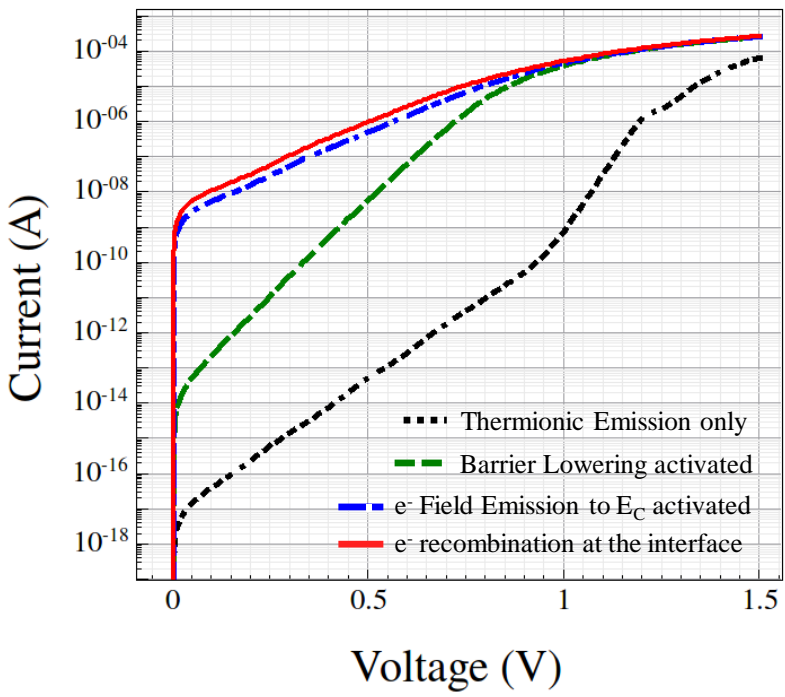

Fig. 9 The significant impact from the inclusion of both the identified bulk traps and interface traps Model A, in Table I, on the carrier transport mechanisms (barrier lowering, recombination at the interface and direct tunnelling) and the simulated sub-threshold current.

The simulated $\log (\mathrm{I})-\mathrm{V}$, in Fig. 10, splits upon regions of influence from the introduced traps, in Table I. These regions illustrate how the various identified trap types in the LSBD play a key role in the fitting process with the increasing forward bias. The subthreshold current is influenced mostly by donor interface traps at lower applied voltage and as the applied voltage increases, the influence from acceptor states increases. In the region between $0-0.5 \mathrm{~V}$, the impact of bulk traps on the total current in the overall $\mathrm{I}-\mathrm{V}$ characteristics is almost negligible. This is because the concentration of interface traps is substantially higher and, as shown in Fig. 7, initially $(\mathrm{Vf}=0)$ all the modelled concentration of the donor interface traps are ionized, shadowing any effect from the bulk traps. With increasing $\mathrm{Vf}>0$ the $\mathrm{E}_{\mathrm{F}}$ movement reduces the amount of these interface traps that are ionized. The closer we get to the on-state the more dominant the bulk ionized traps become mainly due to scattering mechanisms. For larger $\mathrm{Vf}>0$ values, their impact on increases and in the on-state the bulk traps have a dominant effect on the I-V shape. It is assumed at this stage that these traps feature uniform spatial properties along the Schottky contact. Hence, a homogeneous behaviour of the barrier height is modelled taking into account the total effect from both the interfacial and bulk traps. This assumption for homogeneous SBH results in very good prediction accuracy almost along the entire measured data curve, besides the lower voltage level part. This part of the $\log (\mathrm{I})-\mathrm{V}$, highlighted in green shade in Fig. 10, suggests some degree of inhomogeneity of the SBH value across the fabricated contact.

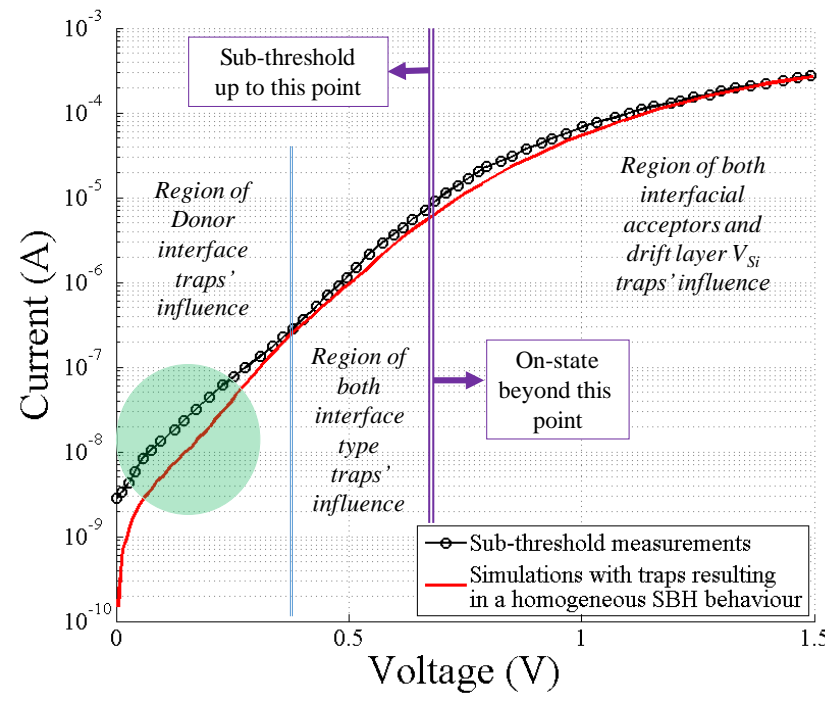

Fig. 10 The incorporation of both the bulk and interface traps model A, in Table I, in the simulations result in a very good prediction of the $\log (\mathrm{I})-\mathrm{V}$ characteristics of the fabricated 3C-SiC-on-Si LSBD. The presented regions of influence are to illustrate where each one of the aforementioned trap distributions play a key role in the fitting process followed.

\section{Interface traps - Model B: Inhomogeneous SBH with additional distribution of states}

The sub-threshold analysis in the previous sub-section indicated that for low forward bias conditions a lateral nonuniformity of the barrier height exists. Such inhomogeneous features are likely due to the large dimensions of the Schottky contacts being studied [22]. The green highlighted part, in Fig. 10 , implies the contribution of additional states at the interface, whose presence creates the conditions for more majority carriers to cross the barrier. These extra states are modelled to act on a limited area of the Schottky contact. To set the fraction of the contact area affected by the inhomogeneity, it was a guided by measurements procedure, as this is a process and material quality dependent parameter. In our case, we achieved the best results with the additional states to feature a spatial distribution within a fraction of $\sim 10 \%$ of the Schottky active area. The properties of these additional interfacial traps were determined with simulations and are presented in Table II as traps' model B. The joint effect from both traps' models, A \& B, determine a region of the Schottky interface prone to conduct larger current for small forward bias values. Within this "weak" region, the SBH can be considered to have a smaller value compared to the rest of the contact, essentially exhibiting an overall non-uniform behavior.

Table II. Additional identified interface traps in the fabricated $\mathrm{Pt} / 3 \mathrm{C}$ $\mathrm{SiC}-\mathrm{on}-\mathrm{Si}$ resulting in a inhomogeneous Schottky contact

\begin{tabular}{|c|c|c|c|c|}
\hline \multirow{2}{*}{\multicolumn{2}{|c|}{ Trap description }} & \multirow{2}{*}{$\begin{array}{c}\text { Type / } \\
\text { Concentration }\end{array}$} & \multicolumn{2}{|c|}{ Distributions } \\
\hline & & & Energetic & Spatial \\
\hline 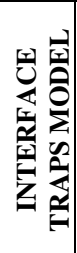 & $\begin{array}{c}\text { Superimposed } \\
\text { Schottky } \\
\text { interface } \\
\text { states to } \\
\text { model the } \\
\text { barrier height } \\
\text { non } \\
\text { uniformity }\end{array}$ & $\begin{array}{c}2 \times 10^{18} \mathrm{~cm}^{-3} \\
\text { of } \\
\text { Donor-like } \\
\text { states }\end{array}$ & $\begin{array}{c}\text { Uniform } \\
\text { Band } \\
E_{\text {Mid }}= \\
0.5 \mathrm{eV} \\
E_{\text {Sig }}= \\
0.2 \mathrm{eV} \\
\left(\text { from } E_{C}^{3 C}\right)\end{array}$ & $\begin{array}{l}\text { Uniform } \\
\text { along only } \\
10 \% \text { of the } \\
\text { contact in } \\
\text { the lateral } \\
\text { direction }\end{array}$ \\
\hline
\end{tabular}

The traps' model B are active mainly at the start of the forward bias conditions. They are a donor-like distribution of interfacial states with the energy levels of these states being 
above the $\left.E_{F}^{e f f}\right|_{I T}$ at zero-bias conditions. In consequence, they are ionized and, thus, highly likely to be occupied with holes. While occupied, they hold a positive charge. The affected region on the Schottky contact will thus experience an enhanced concentration of charged donor-like states due to both traps' distributions (traps of model A + traps of model B). The accumulated positive charge at this region enhances the barrier lowering allowing for more electrons to cross for the same bias and temperature conditions. Further, the induced band bending is stronger increasing the FE.

Fig. 11 depicts how the identified interfacial traps, in Table I and Table II, are able to model a non-uniform SBH along the Schottky contact by featuring different spatial distributions. The X2 point belongs to the assumed weak region where the effect from both traps' model A and B applies. On the other hand, the $\mathrm{X} 1$ point is representative of regions on the contact experiencing the effect from the traps' model A only, corresponding to the band condition in Fig. 8. The dotted $E_{C}$ line in Fig. 11 corresponds to the initial band bending in Fig. 5. The solid $E_{C}$ line indicates the current band bending induced at each point due to the overall effect of the identified traps in Table I and Table II. Finally, the dashed $E_{C}$ line in the inset band diagram at point X2 corresponds to the initial effect from the traps' model A, before the additional traps' model B results in the final solid line realizing the inhomogeneity of the $\mathrm{SBH}$. Although X2 has been modelled to be located at the edge of the device, this is also the boundary of the simulation domain. From the numerical point of view, the equations are solved with the assumption that the device is mirrored at the boundary. So, placing the traps of Model B at the edge or in any position along the Schottky interface does not affect the results. Secondly, the metals have been modelled to have no lateral voltage drop. Hence the simulated current resembles the average total current simulated to flow through the metal. The consequence of all above is that it is the total area covered by traps of Model B that matters and not the exact location.

The energy levels of the donor-like states in traps' model B expand up to the lowest level of the acceptor-like states energetic distribution in model A. This also limits their contribution on a small range of forward bias values. With the increased forward bias, the $\left.E_{F}^{e f f}\right|_{I T}$ will move upwards decreasing the number of the donor-like states that are likely to be occupied by a hole. When the forward bias reaches a sufficient value for the $\left.E_{F}^{\text {eff }}\right|_{I T}$ to ionize the acceptor-like states, specified in the traps' model A, then the effect of the traps' model B will become negligible. Thereafter, the simulated Schottky contact will demonstrate a uniform behaviour of the SBH, as the result of the traps' model A effect only. The developed TCAD model, which incorporates all the trap distributions in Table I and Table II, is able to accurately describe the electrical performance of the fabricated LSBD. The final match achieved between measurements and simulations, in Fig. 12, adds great confidence to the validity of the combined effect from the involved carrier transport mechanisms and the identified traps.

\section{IMPACT OF TEMPERATURE}

At elevated temperature, the total subthreshold current increases. The impact of increased temperature on the individual carrier transport mechanism is analysed in subsection A, whereas the impact of temperature on the importance of inhomogeneities is analysed in subsection B.

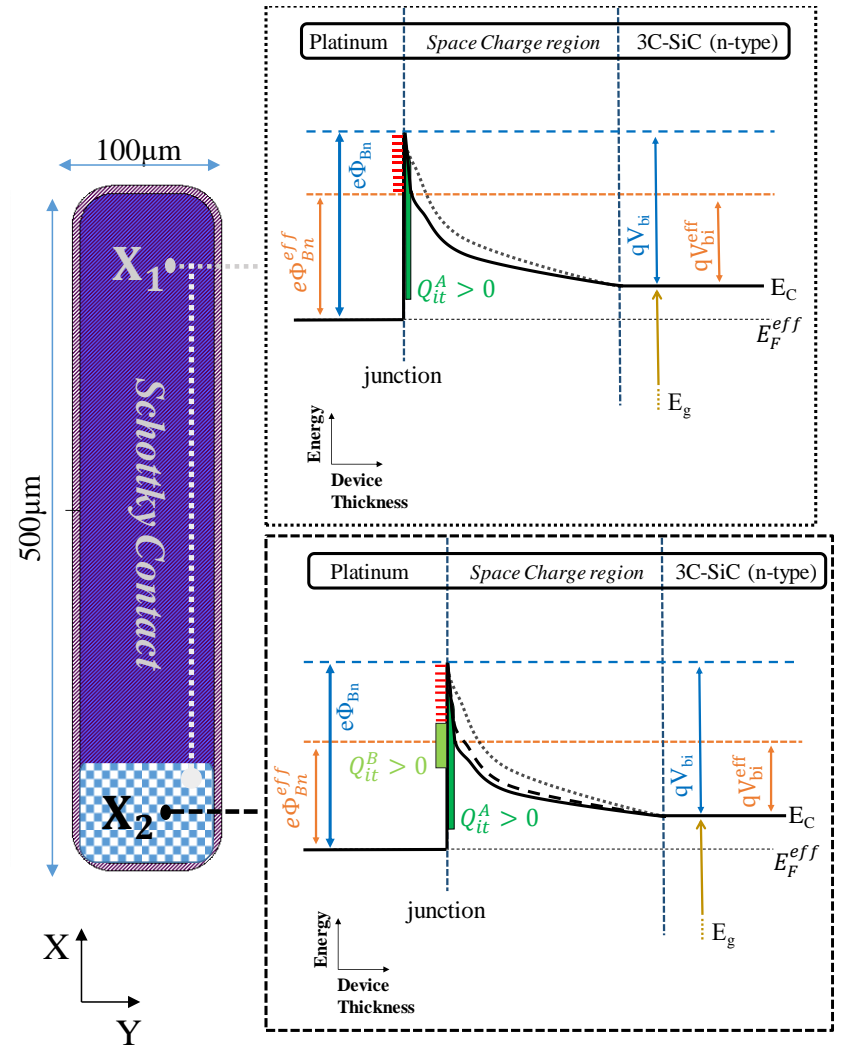

Fig. 11 Inhomogeneous behaviour of the fabricated 3C-SiC-on-Si Schottky contacts. The $\mathrm{X} 1$ point is indicative of a location on the Schottky active area where the interfacial traps' model A is uniformly applied. The $\mathrm{X} 2$ point belongs to a portion of the Schottky active area in which the identified interfacial traps' model A and B spatially overlap. The additive effect of the traps within this portion of the contact forms a region of lower $\mathrm{SBH}$ value.

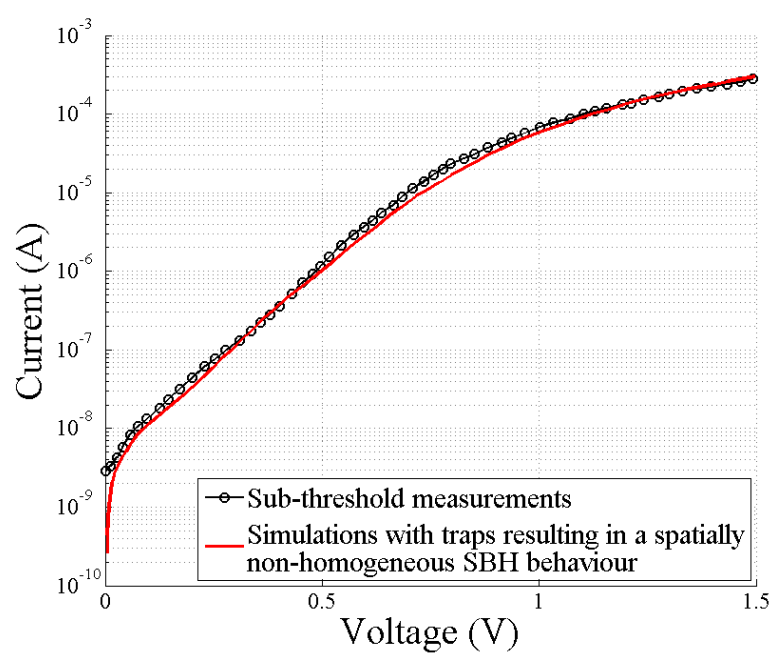

Fig. 12 The proposed TCAD model is able to accurately replicate the electrical performance of the fabricated $\mathrm{Pt} / 3 \mathrm{C}-\mathrm{SiC}$-on-Si lateral Schottky contacts by physically linking the SBH behaviour, including any observed inhomogeneous features, to the effect induced by the identified traps.

\section{A. Impact on individual carrier transport mechanisms.}

In Fig. 13 the impact of elevated temperature on the individual carrier transport mechanisms is demonstrated when interface traps model $\mathrm{A}$ is considered. To understand this, the concept of electrons' occupation distribution should be introduced and analysed. At any given temperature, the kinetic 
energy of the majority carriers defines the shape of the occupation distribution of electrons in the $\mathrm{E}_{\mathrm{C}}$. The latter has been calculated and is illustrated in Fig. 14.

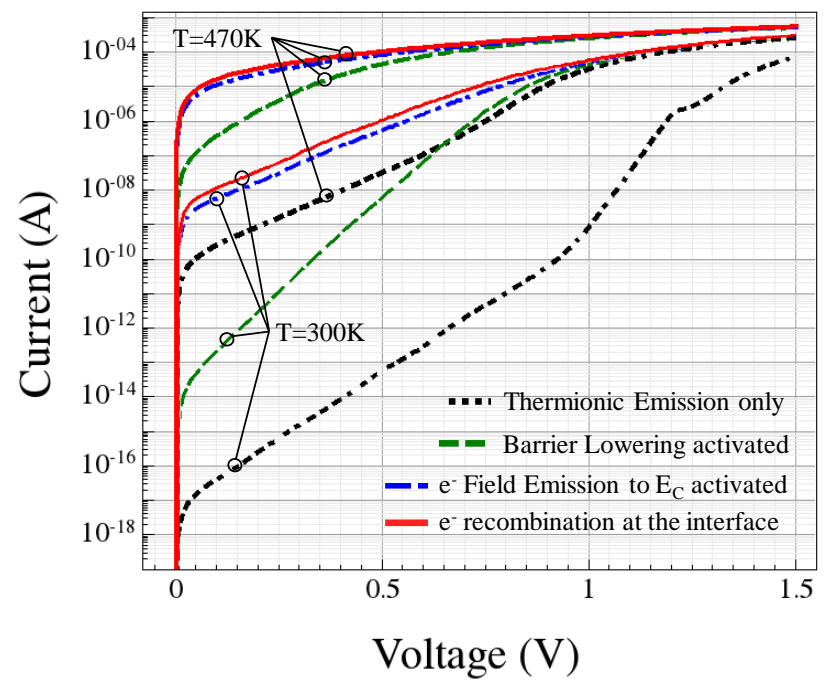

Fig. 13 The impact of temperature on the carrier transport mechanisms (thermionic emission, barrier lowering, direct tunnelling and recombination at the interface). The simulation results at $470 \mathrm{~K}$ are compared with those at $300 \mathrm{~K}$ from Fig. 9 when interface traps model A is considered.

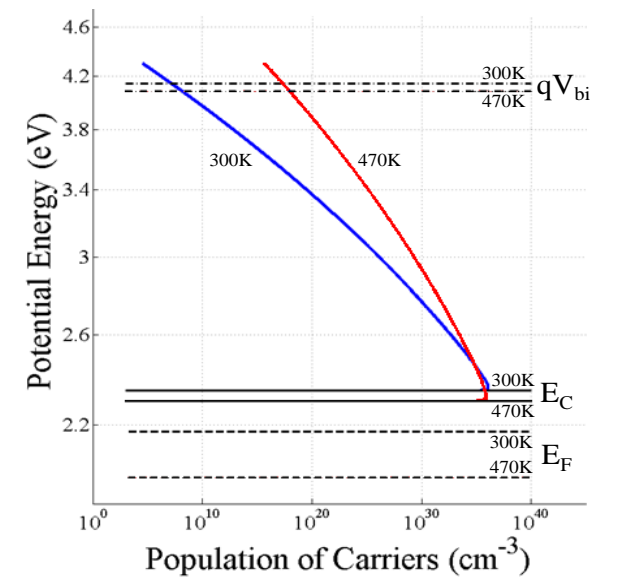

Fig. 14 Occupation distribution of electrons in the Conduction Band of $3 \mathrm{C}-\mathrm{SiC}$ at $300 \mathrm{~K}$ and $400 \mathrm{~K}$. The $\mathrm{y}$-axis is in log-scale.

According to TE theory, the resulting current comprises a component due to majority electrons crossing the barrier from the metal to the semiconductor $\left(\mathrm{J}_{\mathrm{MS}}\right.$ ) and another component due to electrons crossing the barrier from the semiconductor to the metal $\left(\mathrm{J}_{\mathrm{SM}}\right)$. In forward bias the resultant current is expressed as $\mathbf{J}=\mathbf{J}_{\mathrm{SM}}-\mathrm{J}_{\mathrm{MS}}$, whilst under thermal equilibrium conditions $\mathrm{J}_{\mathrm{SM}}=\mathrm{J}_{\mathrm{MS}}=\mathrm{J}_{0}$ applies, where $\mathrm{J}_{0}$ the leakage current. The $J_{S M}$ in (2) is directly linked with the quantity $n_{s}$, which determines the amount of electrons $/ \mathrm{cm}^{3}$ at the semiconductor surface (i.e. $\mathrm{x}=0$ ) with energy higher than the build-in potential barrier $V_{b i}$. The quantity $\bar{V}_{x}$ is the mean velocity component obtained from particle statistics. In the generalized case of forward bias, the equilibrium conditions are disrupted and the build-in potential becomes $V_{b i}^{\prime}=V_{b i}-V_{f}$. In turn, the ratio of electrons' concentration at the surface and at the equilibrium concentration at the bulk $\left(n_{n 0}\right)$ becomes as described in (3), based on the Fermi-Dirac statistics. Furthermore, the $n_{n 0}$ is directly linked with the density of states $\left(N_{C}\right)$ in the $E_{C}$ according to (4). Substituting (4) in (3), the number of electrons capable of crossing the barrier to the metal side via Thermionic Emission is described in (5). In (5), the expression for $n_{s}$ essentially corresponds to the tail part of the occupation distribution of electrons at $E_{C}$.

$$
\begin{gathered}
J_{S M}=q \cdot n_{S} \cdot \bar{V}_{x} \\
\frac{n_{S}}{n_{n 0}}=e^{-\frac{q\left(V_{b i}-V_{f}\right)}{k T}} \\
n_{n 0}=N_{C} \cdot e^{-\frac{E_{C}-E_{F}}{k T}} \\
n_{S}=N_{C} \cdot e^{-\frac{E_{C}-E_{F}+q V_{b i}-q V_{f}}{k T}}=N_{C} \cdot e^{-\frac{\Phi_{B n}}{k T}} \cdot e^{\frac{V_{f}}{V_{T}}}
\end{gathered}
$$

In (6), the Fermi function gives the probability of occupancy for an available energy state and utilizes the information of the $\mathrm{E}_{\mathrm{F}}$ energetic position within the $E_{g}^{3 C-S i C}$ utilizing (1). In our calculations, the $\mathrm{E}_{\mathrm{F}}$ incorporates both the band gap dependency on temperature and the band gap narrowing phenomenon. Although the Fermi function has a finite value in the gap, there is no electron population at those energies. On the contrary, in semiconductors, the DoS for conduction electrons begins at the top of the band gap. Therefore, the calculated $f(T)$ is factored by the number of available energy states on determining how many electrons actually reach the conduction band of $3 \mathrm{C}-\mathrm{SiC}$. The actual population of the conduction band in $3 \mathrm{C}-\mathrm{SiC}$, or the total occupation distribution, is calculated as the product of the DoS in (7) in the corresponding band with the Fermi function (6). The values utilized in the calculations are shown in Table III.

Table III. Values and parameters used for the calculation of occupation distribution.

\begin{tabular}{|c|c|}
\hline Parameter Name & Value \\
\hline Electron mass in 3C-SiC (in units of $\left.\mathrm{m}_{0}\right)$ & 0.35 \\
\hline Electron rest mass $\left[\mathrm{m}_{0}\right](\mathrm{kg})$ & $9.11 \times 10^{-31}$ \\
\hline Planck's constant $[\mathrm{h}]\left(\mathrm{m}^{2} \mathrm{~s}^{-1} \mathrm{~kg}\right)$ & $6.626 \times 10^{-34}$ \\
\hline
\end{tabular}

$$
\begin{gathered}
f(T)=\frac{1}{1+e^{\left(\frac{E-E_{F}}{k T}\right)}} \\
p(E)=\frac{8 \sqrt{2 \cdot \pi} \cdot m^{3 / 2}}{h^{3}} \sqrt{E-E_{g}(T)} \\
\frac{m_{t}(T)}{m_{0}}=\alpha \frac{E_{g}(0)}{E_{g}(T)}
\end{gathered}
$$

For the investigated $\mathrm{Pt} / 3 \mathrm{C}-\mathrm{SiC}$ interface, operation in elevated temperatures suggests that many material parameters change. Considering two temperature values of $\mathrm{T}=300 \mathrm{~K}$ and $\mathrm{T}=470 \mathrm{~K}$, the bandgap value decreases, whilst the electron affinity value slightly increases with temperature. According to the models for 3C-SiC discussed in [45], [46], these values were calculated and are listed in Table IV. In turn, the electrons' barrier linked to the $\mathrm{J}_{\mathrm{SM}}, q V_{b i}=q \Phi_{M}-q X_{3 C}$ will slightly reduce, given $q \Phi_{M}$ maintains its value. According to (1) the $\mathrm{E}_{\mathrm{F}}$ moves energetically closer to $\mathrm{E}_{\mathrm{i}}$ within $E_{g}^{3 C-S i C}$ due to the increased intrinsic carrier concentration at higher temperatures. However, the Fermi function becomes wider, hence overlapping more DoS in the $E_{C}$. At higher temperatures this change in the shape of the Fermi function indicates that more electrons could reach the conduction band and thus contribute to the $\mathrm{J}_{\mathrm{SM}}$.

The DoS, in (7), changes following the $E_{g}(T)$ dependence. For $\mathrm{T}=470 \mathrm{~K}$, a reduced bandgap value suggests a higher density of available energy states at the conduction 
band of 3C-SiC. Thus, at high temperatures, both the increased available density of states and the wider Fermi function in the conduction band impact the shape of the occupation distribution, essentially increasing the conducting population of electrons. This is illustrated in Fig. 13, where the subthreshold current at $\mathrm{T}=300 \mathrm{~K}$ and $\mathrm{T}=470 \mathrm{~K}$ are compared. Notably, for $\mathrm{T}=470 \mathrm{~K}$ the tail of the occupation distribution (shown in Fig 14), which is the part of the distribution beyond the $q V_{b i}$, suggests orders of magnitude more electrons are now capable of crossing this energy barrier by TE. Therefore, it is expected that TE becomes dominant at elevated temperatures, reducing the effect of the considered interface trap profiles on the resulting sub-threshold current. This is in agreement with the simulation results of Fig. 13 where the TE contribution to $\mathrm{J}_{\mathrm{SM}}$ increases by almost seven orders of magnitude at the expense of the BL mechanism. At $\mathrm{T}=300 \mathrm{~K}$, the ionized donor-states at the interface create the conditions for enhanced TFE due to BL. The more the ionized donor states, the greater the resulting BL, and thus, the TFE. On the contrary, for $\mathrm{T}=470 \mathrm{~K}$, the increased population of the electrons at the tail of the occupation distribution shadows the observed extensive contribution of the BL at $\mathrm{T}=300 \mathrm{~K}$. Nonetheless, the wider shape (due to the higher slope) of the occupation distribution for $\mathrm{T}=470 \mathrm{~K}$ compared to the case of $\mathrm{T}=300 \mathrm{~K}$ in Fig. 14, still suggests noticeable BL contribution to the $\mathrm{J}_{\mathrm{SM}}$ due to the ionization of the considered distributions of states at the interface. Further, the simulations indicate that at $\mathrm{T}=470 \mathrm{~K}$ the contribution from $\mathrm{FE}$ and recombination at the interface is slightly reduced compared to operation at $\mathrm{T}=300 \mathrm{~K}$, highly likely due to the tunneling mass of electrons increasing with temperature [58]. The latter is described in (9) [59], where $0<\alpha<1$, taking into account the decreasing $3 \mathrm{C}-\mathrm{SiC}$ energy gap values with temperature. An increased tunneling mass reduces, in turn, the probability for the corresponding carrier to FE [60]. Overall, the effect of the traps on the carrier transport mechanisms is still apparent, yet largely reduces when the SBD operates at elevated temperatures.

Table IV. The effect on temperature on basic 3C-SiC material parameter values

\begin{tabular}{|c|c|c|}
\hline 3C-SiC parameter & $\mathbf{3 0 0 K}$ & $\mathbf{4 7 0 K}$ \\
\hline Bandgap $-E_{g}(T)$ & $2.345 \mathrm{eV}$ & $2.3 \mathrm{eV}$ \\
\hline Electron affinity $-\chi(T)$ & $3.855 \mathrm{eV}$ & $3.8772 \mathrm{eV}$ \\
\hline Intrinsic carrier concentration $-n_{i}(T)$ & $0.228 \mathrm{~cm}-3$ & $1.223 \mathrm{e} 7 \mathrm{~cm}-3$ \\
\hline
\end{tabular}

\section{B. Impact on inhomogeneity}

To study the impact of temperature on the importance of inhomogeneities on the subthreshold current, model B which incorporates an inhomogeneous SBH has been used. Fig 15 shows how the subthreshold current varies with increasing temperature, at $\mathrm{T}=300 \mathrm{~K}, 400 \mathrm{~K}$ and $470 \mathrm{~K}$, with and without the inhomogeneous features. As shown, the effect of inhomogeneity of the Schottky contact becomes weaker when temperature increases. Since the non-uniformity of the barrier height is modeled with a superimposed distribution of states at the interface, this observation is in agreement with the prior interpretation on the effect of the traps on the resulting $\mathrm{J}_{\mathrm{SM}}$.

\section{CONCLUSIONS}

In this paper the fabrication of lateral 3C-SiC-on-Si SBDs allowed to investigate the origins of the leaky behaviour in such devices. In this context, the role of traps is highlighted as

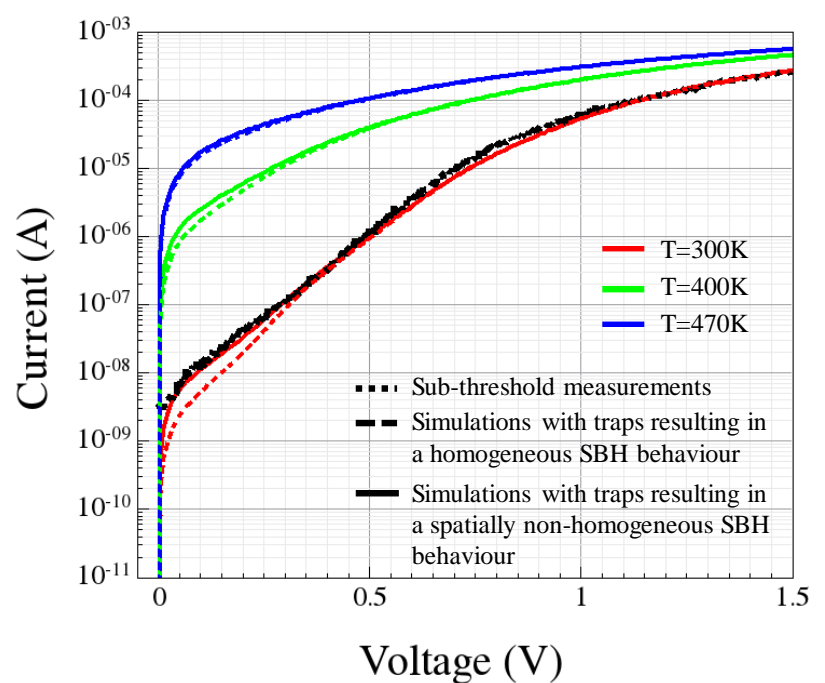

Fig. 15 The impact of temperature on the modelled non-homogeneous behavior of the SBH.

the source of the observed large leakage current, which otherwise cannot be explained by the native WBG properties of the 3C-SiC semiconductor. An advanced TCAD model is proposed to link the significant degree of non-ideality with the existing traps by engaging complex trapping/de-trapping phenomena. Initially, bulk traps of acceptor type have been identified in the 3C-SiC-on-Si through simulations, with activation energy below the effective Fermi level. These deep acceptor levels resemble the intrinsic $V_{S i}$ defects, introduced in $3 \mathrm{C}$-SiC-on-Si due to the out-diffusion of $\mathrm{Si}$ atoms to form the $\mathrm{SiC}$ layer. Subsequently, it was established that the shape of the sub-threshold region of the $\log (\mathrm{I})-\mathrm{V}$ from the characterized LSBD is mainly affected by Schottky interfacial states. The utilization of band diagrams has been deployed to shed light on the effect of these states and, thereafter, their energetic distribution properties were determined through extensive simulations. An interfacial traps' profile was defined, of both donor and acceptor-like states, of amphoteric nature within a limited range of energies, featuring a spatially uniform distribution over the Schottky active area.

The suggested method further enabled for the SBH inhomogeneity interpretation. It was found that a distinct distribution of donor-like states spatially limited in a small portion of the Schottky active area also exists. In turn, this portion of the Schottky contact experiences an additive effect from both the uniform interfacial traps' profile and the distinct donor-like distribution emulating a lower SBH region. Moreover, the simulations revealed that the additional donorlike distribution of states should be energetically expanding in a narrower band of energies at the Schottky interface. Consequently, this results in an inhomogeneous overall behaviour of the Schottky contact with forward bias. After the inclusion of all identified traps, both bulk and interfacial, the TCAD model achieved very good prediction of the LSBD I$\mathrm{V}$ characteristics which match the measurements with excellent accuracy.

The effect of temperature was thereafter assessed with simulations, engaging the information of the identified trap profiles. It was found that the impact of the interface traps as well as the $\mathrm{SBH}$ inhomogeneity reduce with temperature. This observation was verified after a physics-based analysis which included calculations of the occupation distribution of electrons in the $\mathrm{E}_{\mathrm{C}}$ of $3 \mathrm{C}-\mathrm{SiC}$. The tail of the occupation 
distribution, determining the population of conductive electrons becomes larger at higher temperatures, resulting in TE dominating the sub-threshold current. Nonetheless, the contribution of the identified trap profiles is still considerable at elevated temperatures due to the smaller gradient of the occupation distribution.

The suggested model and analysis methodology is not limited to 3C-SiC-on-Si, rather it can be applied to any Metal / Semiconductor junction to elucidate the effect from the inevitable presence of traps on the SBH and the electrical performance of the device.

\section{REFERENCES}

[1] J. Millan, P. Godignon, X. Perpina, A. Perez-Tomas, and J. Rebollo, "A Survey of Wide Bandgap Power Semiconductor Devices," IEEE Trans. Power Electron., vol. 29, no. 5, pp. 2155-2163, 2014. doi:10.1109/TPEL.2013.2268900

[2] A. Elasser and T. P. Chow, "Silicon carbide benefits and advantages for power electronics circuits and systems," Proc. IEEE, vol. 90, no. 6, pp. 969-986, 2002. doi:10.1109/JPROC.2002.1021562

[3] T. P. Chow, "High-voltage $\mathrm{SiC}$ and $\mathrm{GaN}$ power devices," Microelectron. Eng., vol. 83, no. 1, pp. 112-122, 2006. doi:10.1016/j.mee.2005.10.057

[4] J. L. Hudgins, G. S. Simin, E. Santi, and M. A. Khan, "An assessment of wide bandgap semiconductors for power devices," IEEE Trans. Power Electron., vol. 18, no. 3, pp. 907-914, 2003. doi:10.1109/TPEL.2003.810840

[5] M. Kanechika, T. Uesugi, and T. Kachi, "Advanced SiC and GaN power electronics for automotive systems," in Technical Digest International Electron Devices Meeting, IEDM, 2010. doi:10.1109/IEDM.2010.5703356, pp. 324-327

[6] G. Ferro, "3C-SiC Heteroepitaxial Growth on Silicon: The Quest for Holy Grail,” Crit. Rev. Solid State Mater. Sci., vol. 40, no. 1, pp. 56-76, 2015. doi:10.1080/10408436.2014.940440

[7] F. La Via, A. Severino, R. Anzalone, C. Bongiorno, G. Litrico, M. Mauceri, M. Schoeler, P. Schuh, and P. Wellmann, "From thin film to bulk 3C-SiC growth: Understanding the mechanism of defects reduction," Mater. Sci. Semicond. Process., vol. 78, pp. 57-68, 2018. doi:10.1016/j.mssp.2017.12.012

[8] H. Nagasawa, K. Yagi, T. Kawahara, and N. Hatta, "Properties of Free-Standing 3C-SiC Monocrystals grown on Undulant- Si ( 001 ) Substrate," vol. $\quad 436, \quad$ pp. $\quad 3-8, \quad 2003$. doi:10.4028/www.scientific.net/MSF.433-436.3

[9] K. Sasaki, E. Sakuma, S. Misawa, S. Yoshida, and S. Gonda, "Hightemperature electrical properties of $3 \mathrm{C}-\mathrm{SiC}$ epitaxial layers grown by chemical vapor deposition," Appl. Phys. Lett., vol. 45, no. 1, pp. 72-73, 1984. doi:10.1063/1.94973

[10] A. A. Lebedev, S. P. Lebedev, V. Y. Davydov, S. N. Novikov, and Y. N. Makarov, "Growth and investigation $\mathrm{SiC}$ based heterostructures," in 15th Biennial Baltic Electronics Conference (BEC), 2016. doi:10.1109/BEC.2016.7743717, pp. 5-6

[11] O. Bouketir, "Advances and Challenges in WBG Devices and their Applications in Power Conversion and Conditioning," in Proceedings of the International Conference on Recent Advances in Electrical Systems, 2016, pp. $34-40$

[12] S. Roy, C. Jacob, and S. Basu, "Current transport properties of Pd/3CSiC Schottky junctions with planar and vertical structures," Solid State Sci., vol. 6, no. 4, pp. 377-382, 2004. doi:10.1016/j.solidstatesciences.2004.01.003

[13] A. Severino, G. D’Arrigo, C. Bongiorno, S. Scalese, F. La Via, and G. Foti, "Thin crystalline 3C-SiC layer growth through carbonization of differently oriented Si substrates," J. Appl. Phys., vol. 102, no. 2, 2007. doi:10.1063/1.2756620

[14] A. Stefanskyi, Ł. Starzak, and A. Napieralski, "Silicon Carbide Power Electronics for Electric Vehicles," 2015 Tenth Int. Conf. Ecol. Veh. Renew. Energies, pp. 1-9, 2015. doi:10.1109/EVER.2015.7138047

[15] G. Colston, S. D. Rhead, V. A. Shah, O. J. Newell, I. P. Dolbnya, D. R. Leadley, and M. Myronov, "Mapping the strain and tilt of a suspended 3C-SiC membrane through micro X-ray diffraction," Mater. Des., vol. 103, pp. 244-248, 2016. doi:10.1016/j.matdes.2016.04.078

[16] Y. Li, Z. Zhao, L. Yu, Y. Wang, Z. Yin, Z. Li, and P. Han, "Heteroepitaxial 3C-SiC on Si ( 100 ) with flow-modulated carbonization process conditions," J. Cryst. Growth, vol. 506, no. September 2018, pp. 114-116, 2019. doi:10.1016/j.jcrysgro.2018.09.037

[17] X. Song, J. F. Michaud, F. Cayrel, M. Zielinski, M. Portail, T. Chassagne, E. Collard, and D. Alquier, "Evidence of electrical activity of extended defects in 3C-SiC grown on Si," Appl. Phys. Lett., vol. 96, no. 14, pp. 1-4, 2010. doi:10.1063/1.3383233

[18] P. Hens, G. Wagner, A. Hölzing, R. Hock, and P. Wellmann, "Dependence of the seed layer quality on different temperature ramp-up conditions for 3C-SiC hetero-epitaxy on Si (100)," Thin Solid Films, vol. 522, pp. 2-6, 2012. doi:10.1016/j.tsf.2011.10.177

[19] S. R. Nutt, D. J. Smith, H. J. Kim, and R. F. Davis, "Interface structures in beta-silicon carbide thin films," Appl. Phys. Lett., vol. 50, no. 4, pp. 203-205, 1987. doi:10.1063/1.97661

[20] F. Giannazzo, G. Greco, S. Di Franco, P. Fiorenza, I. Deretzis, A. La Magna, C. Bongiorno, M. Zimbone, F. La Via, M. Zielinski, and F. Roccaforte, "Impact of Stacking Faults and Domain Boundaries on the Electronic Transport in Cubic Silicon Carbide Probed by Conductive Atomic Force Microscopy," Adv. Electron. Mater., vol. 6, no. 2, pp. 1-8, 2020. doi:10.1002/aelm.201901171

[21] W. Monch, "On the physics of metal-semiconductor interfaces," Reports Prog. Phys., vol. 53, no. 3, pp. 221-278, Mar. 1990. doi:10.1088/0034-4885/53/3/001

[22] J. Eriksson, M. H. Weng, F. Roccaforte, F. Giannazzo, S. Leone, and V. Raineri, "Toward an ideal Schottky barrier on 3C -SiC," Appl. Phys. Lett., vol. 95 , no. 8, 2009. doi:10.1063/1.3211965

[23] R. T. Tung, "Electron transport at metal-semiconductor interfaces: General theory," Phys. Rev. B, vol. 45, no. 23, pp. 13509-13523, 1992

[24] J. H. Werner and H. H. Güttler, "Barrier inhomogeneities at Schottky contacts," J. Appl. Phys., vol. 69, no. 3, pp. 1522-1533, 1991. doi:10.1063/1.347243

[25] S. U. Omar, T. S. Sudarshan, T. A. Rana, H. Song, and M. V. S. Chandrashekhar, "Large barrier, highly uniform and reproducible Ni-Si/4H$\mathrm{SiC}$ forward Schottky diode characteristics: Testing the limits of Tung's model," J. Phys. D. Appl. Phys., vol. 47, no. 29, 2014. doi:10.1088/00223727/47/29/295102

[26] J. L. Li, Y. Li, L. Wang, Y. Xu, F. Yan, P. Han, and X. L. Ji, "Influence of deep defects on electrical properties of Ni/4H-SiC Schottky diode," Chinese Phys. B, vol. 28, no. 2, 2019. doi:10.1088/1674-1056/28/2/027303

[27] D. Defives, O. Noblanc, C. Dua, C. Brylinski, M. Barthula, and F. Meyer, "Electrical characterization of inhomogeneous $\mathrm{Ti} / 4 \mathrm{H}-\mathrm{SiC}$ Schottky contacts," Mater. Sci. Eng. R Reports, vol. 62, pp. 395-401, 1999. doi:10.1016/S0921-5107(98)00541-8 
[28] B. J. Skromme, E. Luckowski, K. Moore, M. Bhatnagar, C. E. Weitzel, T. Gehoski, and D. Ganser, "Electrical characteristics of Schottky barriers on 4H-SiC: The effects of barrier height nonuniformity," J. Electron. Mater., vol. 29, no. 3, pp. 376-383, 2000. doi:10.1007/s11664-000-0081-9

[29] I. Nikitina, K. Vassilevski, A. Horsfall, N. Wright, A. G. O’Neill, S. K. Ray, K. Zekentes, and C. M. Johnson, "Phase composition and electrical characteristics of nickel silicide Schottky contacts formed on $4 \mathrm{H}-\mathrm{SiC}$," Semicond. Sci. Technol., vol. 24, no. 5, 2009. doi:10.1088/02681242/24/5/055006

[30] X. Ma, P. Sadagopan, and T. S. Sudarshan, "Investigation on barrier inhomogeneities in 4H-SiC Schottky rectifiers," Phys. Status Solidi Appl. Mater. Sci., vol. 203, no. 3, pp. 643-650, 2006. doi:10.1002/pssa.200521017

[31] P. M. Gammon, E. Donchev, A. Pérez-Tomás, V. A. Shah, J. S. Pang, P. K. Petrov, M. R. Jennings, C. A. Fisher, P. A. Mawby, D. R. Leadley, and N. McN. Alford, "A study of temperature-related non-linearity at the metalsilicon interface," J. Appl. Phys., vol. 112, no. 11, 2012. doi:10.1063/1.4768718

[32] V. Kumar, A. S. Maan, and J. Akhtar, "Barrier height inhomogeneities induced anomaly in thermal sensitivity of $\mathrm{Ni} / 4 \mathrm{H}-\mathrm{SiC}$ Schottky diode temperature sensor," J. Vac. Sci. Technol. B, Nanotechnol. Microelectron. Mater. Process. Meas. Phenom., vol. 32, no. 4, p. 041203, 2014. doi:10.1116/1.4884756

[33] M. E. Aydin, N. Yildirim, and A. Türüt, "Temperature-dependent behavior of Ni/4H-nSiC Schottky contacts," J. Appl. Phys., vol. 102, no. 4, 2007. doi:10.1063/1.2769284

[34] G. S. Chung, K. S. Kim, and F. Yakuphanoglu, "Electrical characterization of $\mathrm{Au} / 3 \mathrm{C}-\mathrm{SiC} / \mathrm{n}-\mathrm{Si} / \mathrm{Al}$ Schottky junction," J. Alloys Compd., vol. 507, no. 2, pp. 508-512, 2010. doi:10.1016/j.jallcom.2010.08.004

[35] R. T. Tung, "Electron transport at metal-semiconductor interfaces: General theory," Phys. Rev. B, vol. 45, no. 23, pp. 13509-13523, 1992. doi:10.1103/PhysRevB.45.13509

[36] R. T. Tung, "Recent advances in Schottky barrier concepts," Mater. Sci. Eng. R Reports, vol. 35, no. 1-3, pp. 1-138, 2001. doi:10.1016/S0927796X(01)00037-7

[37] J. P. Sullivan, R. T. Tung, M. R. Pinto, and W. R. Graham, "Electron transport of inhomogeneous Schottky barriers : A numerical study," J. Appl. Phys., vol. 70, no. 12, pp. 7403-7424, 1991. doi:10.1063/1.349737

[38] H.-J. Im, Y. Ding, J. P. Pelz, and W. J. Choyke, "Nanometer-scale test of the Tung model of Schottky-barrier height inhomogeneity," Phys. Rev. B vol. 64, no. 7, p. 075310, 2001. doi:10.1103/PhysRevB.64.075310

[39] F. La Via, M. Camarda, and A. La Magna, "Mechanisms of growth and defect properties of epitaxial SiC," Appl. Phys. Rev., vol. 1, no. 3, 2014. doi:10.1063/1.4890974

[40] Y. Yamamoto, S. Harada, K. Seki, A. Horio, T. Mitsuhashi, D. Koike, M. Tagawa, and T. Ujihara, "Low-dislocation-density 4H-SiC crystal growth utilizing dislocation conversion during solution method," Appl. Phys. Express, vol. 7, no. 6, 2014. doi:10.7567/APEX.7.065501

[41] M. Benamara, M. Anani, B. Akkal, and Z. Benamara, "Ni/SiC-6H Schottky Barrier Diode interfacial states characterization related to temperature," J. Alloys Compd., vol. 603, pp. 197-201, 2014. doi:10.1016/j.jallcom.2014.02.177

[42] K. Y. Lee and Y. H. Huang, "An investigation on barrier inhomogeneities of $4 \mathrm{H}-\mathrm{SiC}$ Schottky barrier diodes induced by surface morphology and traps," IEEE Trans. Electron Devices, vol. 59, no. 3, pp. 694-699, 2012. doi:10.1109/TED.2011.2181391

[43] T. Katsuno, Y. Watanabe, H. Fujiwara, M. Konishi, H. Naruoka, J. Morimoto, T. Morino, and T. Endo, "Analysis of surface morphology at leakage current sources of $4 \mathrm{H}-\mathrm{SiC}$ Schottky barrier diodes," Appl. Phys. Lett., vol. 98, no. 22, pp. 2-5, 2011. doi:10.1063/1.3597413

[44] K. Y. Lee and M. A. Capano, "The correlation of surface defects and reverse breakdown of 4H-SiC Schottky barrier diodes," J. Electron. Mater., vol. 36, no. 4, pp. 272-276, 2007. doi:10.1007/s11664-006-0075-3

[45] A. Arvanitopoulos, N. Lophitis, K. N. Gyftakis, S. Perkins, and M. Antoniou, "Validated physical models and parameters of bulk 3C-SiC aiming for credible technology computer aided design (TCAD) simulation," Semicond. Sci. Technol., vol. 32, no. 10, p. 104009, 2017. doi:10.1088/13616641/aa856b

[46] N. Lophitis, A. Arvanitopoulos, S. Perkins, and M. Antoniou, "TCAD Device Modelling and Simulation of Wide Bandgap Power Semiconductors," in Disruptive Wide Bandgap Semiconductors, Related Technologies, and Their Applications, Y. K. Sharma, Ed. Rijeka: InTech, 2018

[47] F. Li, Y. Sharma, V. Shah, M. Jennings, A. Pérez-Tomás, M. Myronov, C. Fisher, D. Leadley, and P. Mawby, "Electrical activation of nitrogen heavily implanted 3C-SiC(1 0 0)," Appl. Surf. Sci., vol. 353, no. October 2015, pp. 958-963, 2015. doi:10.1016/j.apsusc.2015.06.169

[48] Keysight, "Keysight B1505A Power Device Analyzer/Curve Tracer." 2011

[49] T. J. Drummond, "Work Functions of the Transition Metals and Metal Silicides," Albuquerque, 1999

[50] C. Kenney, K. C. Saraswat, B. Taylor, and P. Majhi, "Thermionic Field Emission Explanation for Nonlinear Richardson Plots," IEEE Trans. Electron Devices, vol. 58, no. 8, pp. 2423-2429, Aug. 2011. doi:10.1109/TED.2011.2156411

[51] M. Mandurrino, G. Verzellesi, M. Goano, M. E. Vallone, F. Bertazzi, G. Ghione, M. Meneghini, G. Meneghesso, and E. Zanoni, "Trap-assisted tunneling in InGaN/GaN LEDs: Experiments and physics-based simulation," Proc. Int. Conf. Numer. Simul. Optoelectron. Devices, NUSOD, pp. 13-14, 2014. doi:10.1109/NUSOD.2014.6935332

[52] B. Asllani, M. Berthou, D. Tournier, P. Brosselard, and P. Godignon, "Modeling of Inhomogeneous 4H-SiC Schottky and JBS Diodes in a Wide Temperature Range," Mater. Sci. Forum, vol. 858, pp. 741-744, 2016

[53] L. Wenchang, Z. Kaiming, and X. Xide, "An electronic structure study of single native defects in beta -SiC," J. Phys. Condens. Matter, vol. 5, no. 7, pp. 891-898, Feb. 1993. doi:10.1088/0953-8984/5/7/016

[54] A. Arvanitopoulos, F. Li, M. R. Jennings, S. Perkins, K. N. Gyftakis, M. Antoniou, P. Mawby, and N. Lophitis, "Experimental Investigation and Verification of Traps affecting the performance of 3C-SiC-on-Si Schottky Barrier Diodes," in IEEE Energy Conversion Congress and Exposition (ECCE), 2019. doi:10.1109/ECCE.2019.8912232, pp. 1941-1947

[55] A. Arvanitopoulos, M. Antoniou, M. R. Jennings, S. Perkins, K. N. Gyftakis, P. Mawby, and N. Lophitis, "A Defects-Based Model on the Barrier Height Behavior in 3C-SiC-on-Si Schottky Barrier Diodes," IEEE J. Emerg. Sel. Top. Power Electron., vol. 8, no. 1, pp. 54-65, 2020. doi:10.1109/JESTPE.2019.2942714

[56] M. Walters, "Radiation-Induced Neutral Electron Trap Generation in Electrically Biased Insulated Gate Field Effect Transistor Gate Insulators," J. Electrochem. Soc., vol. 138, no. 9, p. 2756, 1991. doi:10.1149/1.2086050

[57] F. Jazaeri, C. M. Zhang, A. Pezzotta, and C. Enz, "Charge-based modeling of radiation damage in symmetric double-gate MOSFETs," IEEE J. Electron Devices Soc., vol. 6, no. 1, pp. 85-94, 2018. doi:10.1109/JEDS.2017.2772346

[58] O. A. Salvado, B. Asllani, C. Buttay, C. Raynaud, and H. Morel, "Extraction of the $4 \mathrm{H}-\mathrm{SiC} / \mathrm{SiO}$ Barrier Height Over Temperature," IEEE 
Trans. Electron Devices, vol. 67, no. 1, pp. 63-68, 2019. doi:ff10.1109/TED.2019.295518

[59] M. A. Green, "Intrinsic concentration, effective densities of states, and effective mass in silicon,” J. Appl. Phys., vol. 67, no. 6, pp. 2944-2954, 1990. doi:doi.org/10.1063/1.345414

[60] D. A. Neamen, Semiconductor Physics and Devices: Basic Principles, 4th ed. New York: NY: McGraw-Hill, 2012 